\title{
MINUTES OF ANNUAL GENERAL MEETING CANADIAN ASSOCIATION OF TEACHERS OF TECHNICAL WRITING
}

\author{
Sunday May 29, 1988
}

1. The President called the meeting to order at 16:05. Eighteen CATTW members were present.

2. The members approved the agenda for the 1988 AGM and the minutes of the 1987 AGM (as they appeared in Technostyle, summer issue).

3. The President (Jacqueline Bosse-Andrieu) reported that:

- The Society was grateful to SSHRCC for providing money to cover the travel expenses of eight of the conference speakers.

- This was a conference at which East and West truly did meet, since there were speakers and delegates from British Columbia, Alberta, Manitoba, Ontario, Quebec, and New Brunswick.

She expressed personal thanks to the conference committee members, especially to Michael Jordan, for helping her arrange such an excellent programme, to Bruce Lundgren for making top-notch local arrangements, and to Juanita Giesbrecht for her hard work as new Secretary-Treasurer.

4. The vice President (Michael Jordan) encouraged speakers who are planning to submit papers for the 1989 conference at Laval University in Quebec City to include a paragraph demonstrating that their paper represents scholarly endeavours, since such statements would help convince SSHRCC to provide funds to assist with travel expenses for those who could not otherwise be funded.

5. The Past President (Ron Blicq) congratulated the current executive on a job well done. 
6. The Secretary-Treasurer (Ron Blicq speaking for Juanita Giesbrecht) reported that:

1. The bank balance on May 27,1988 was $\$ 2260.39$.

2. Only 10 paid memberships for 1988 have been received to date.

3. A letter dated May 27 will be mailed to all members, asking those whose dues have not been paid to submit them.

7. The Editor of Technostyle (Jennifer Connor) reported that:

- The most recent edition of Technostyle was mailed in May 1988.

- The new format for Technostyle is proving considerably less expensive to produce.

- More articles and papers are needed to maintain both a consistent size for each issue and frequency of publication.

- Anne Parker (University of Manitoba) indicated her willingness to become Associate Editor.

Jennifer suggested that only two (larger) journals be issued in 1989 , and a newsletter be started within the next year. The rationale would be to maintain the identity of Technostyle as a scholarly refereed journal, and to let the Newsletter carry announcements, conference programmes, and more general information. The consensus of those present seemed to be that this would be a worthwhile approach.

Jennifer expressed her thanks to the Editorial Committee for refereeing journal articles. The President also expressed her thanks to the University of Western Ontario for the university's continued assistance in publishing Technostyle.

8. The Secretary-Treasurer (Ron Blicq for Juanita Giesbrecht) chaired the nominations for and appointments to the following committees, for two-year terms:

$\begin{array}{ll}\text { Programme: } & \begin{array}{l}\text { Joan Pavelich (Ottawa) } \\ \text { Lilita Rodman (Vancouver) }\end{array} \\ \text { Nominations: } & \begin{array}{l}\text { Jean Dohaney (Fredericton) } \\ \text { Janet Giltrow (Burnaby, B.C.) }\end{array}\end{array}$


9. The Co-ordinator of the 1989 Conference Programme Committee (Michael Jordan) suggested possible themes for sections of next year's programme: writing with computers; oral presentations; and using non-sexist language. He also will be looking for recommendations from the membership for two guest lecturers, from the U.S.A., Britain, or possibly Europe.

10. The members agreed to disband the committee investigating the "Asian" connection.

11. The members established a committee to consider carrying out a second survey of the Canadian "teaching of technical writing" scene, composed of Louise Lariviere (confirmed), Hyacinth Harewood (to be approached), and one other (to be named). The focus should be on what is being done, what is being taught, where technical writing is being taught, and what new courses are planned.

12. The members discussed the appropriateness of the word "Technical" in the Society's name, but reached no conclusion.

13. The "first choice" dates for the 1989 conference were selected to be Sunday and Monday May 28 and 29. The President will try to align the dates with those selected by the Association of Canadian University Teachers of English (ACUTE) and the Canadian Association for Translation Studies (CATS).

14. The President expressed appreciation for the printing assistance provided to the Society by the University of Ottawa and Red River Community College.

15. The meeting adjourned at $17: 15$.

Jacqueline Bossé-Andrieu

Ron S. Blicq

President CATTW

Acting Secretary, CATTW 


\section{PROCES-VERBAL DE L'ASSEMBLEE GENERALE ANNUELLE DE L'ASSOCIATION CANADIENNE DES PROFESSEURS DE REDACTION TECHINIQUE ET SCIENTIFIQUE QUI S'EST TENUE LE 29 MAI 1988 ì 16 HEURES À L'UNIVERSITE DE WINDSOR}

1. La présidente ouvre la séance $16 \mathrm{~h} 05$. Dix-huit membres sont presents.

2. - L'ordre du jour de la réunion est approuvé.

- Le procès-verbal de la dernière réunion (1987) est approuvé (il avait paru dans le numéro d'été de Technostyle).

3. Rapport de la présidente:

- L'ACPRTS a obtenu, cette année, une aide financiere du Conseil de recherches en sciences humaines. L'Association remercie le CRSHC pour cette subvention qui a couvert les frais de déplacement de 8 conférenciers.

- Le congres de 1988 , selon les voeux exprimés en 1987, a réussi a rassembler des conférenciers et des délégués venus de tout le Canada: Colombie-Britannique, Alberta, Manitoba, Ontario, Québec et Nouveau-Brunswick.

La président remercie a) les membres du comité chargé du congrès, et en particulier Michael Jordan, de l'avoir aidá a élaborer un excellent programme; b) Bruce Lundgren de s'être acquitté de ses tâches de coordinateur local avec autant de brio; c) Juanita Giesbrecht, secrétaire-trésorière, dont la tâche a été particulièrement ingrate au début de son mandat.

4. Rapport du vice-président:

Michael Jordan rappelle qu'il est important que les conférenciers qui envisagent de présenter une communication a l'Université Laval en 1989 incluent, dans leur résumé, un paragraphe montrant que leurs travaux représentent une contribution a l'avancement de la 
recherche au Canada; en effet, l'ACPRTS doit convaincre le CRSHC de l'apport du colloque a "l'activité scientifique" du Canada pour esperer recevoir des fonds qui couvriront les frais de voyage des conférenciers qui ne reçoivent pas d'autre aide financiére.

5. Rapport de l'ex-président:

Ron Blicq félicite le Bureau actuel pour le travail accompli cette année.

6. Rapport de la secétaire-trésorière:

Ron Blicq, prenant la parole au nom de Juanita Giesbrecht, mentionne que:

1. Le solde en banque, le 27 mai 1988, était de 2260.39s;

2. Les cotisations de 1988 n'ont été acquittées que par 10 membres;

3. Une lettre en date du 27 mai sera envoyé a tous les membres demandant a ceux qui n'ont pas encore versé leur cotisation de le faire sans tarder.

7. Rapport de la rédactrice de Technostyle:

Jennifer Connor signale que:

- Le dernier numéro de Technostyle a été posté en mai 1988;

- Gráce à la nouvelle présentation de la revue, les coúts de production de Technostyle ont été considérablement réduits;

- Pour que Technostyle continue a paraftre a une fréquence raisonnable et pour que les numéros aient un volume satisfaisant, la rédactrice a besoin de recevoir des articles;

- Anne Parker (Universite du Manitoba) a signifié son intention d'accepter le poste de rédactrice adjointe.

J. Connor propose qu'en 1989 paraissent seulement deux numéros (plus importants que d'habitude) de Technostyle et qu'un bulletin 
commence a paraitre l'an prochain. De cette façon, Technostyle resterait une revue savante avec comite de rédaction tandis que le bulletin communiquerait les annonces, les programmes des conférences et des renseignements généraux. Cette suggestion semble recueillir l'approbation des membres.

La rédactrice remercie les membres du comité de rédaction d'avoir examiné les articles soumis à la revue. La présidente remercie l'Université de Western Ontario pour l'aide apportée a la publication de Technostyle.

8. Elections:

Ron Blicq (remplaçant la secrétaire-trésorière, Juanita Giesbrecht) préside les élections. Sont élus, pour un mandat de 2 ans:

\section{Comité du programme: Joan Pavelich (Ottawa) Lilita Rodman (Vancouver) \\ Comité des candidatures: Jean Dohaney (Fredericton) Janet Giltrow (Burnaby, C.B.)}

9. Le président du comité du programme du congrès de 1989 (Michael Jordan) propose plusieurs themes sur lesquels pourraient porter certaines séances: rédaction et ordinateurs, présentations orales, l'emploi d'un langage non sexiste. Il envisage aussi la possibilité d'inviter deux conférenciers étrangers (E.-U., G.-B. ou Europe) que lui recommanderont les membres de l'ACPRTS.

10. L'assemblée est d'accord pour dissoudre le comité chargé de la "filière asiatique".

11. L'assemblée établit un comité chargé de mener une deuxième enquête sur "l'enseignement de la rédaction technique" au Canada; ce comité est composé de Louise Larivière et de deux autres membres non nommés (le nom de Hyacinth Harewood est proposé). L'enquête cherchera a savoir ce qui se fait et ce qui s'enseigne, les établissements où la rédaction "technique" (au sens large du terme) s'enseigne et les nouveaux cours et programmes prévus.

12. L'assemblée discute de la pertinence de l'adjectif "technical" dans le nom de l'association. Aucune conclusion n'est atteinte. 
13. Date du congrès de 1989:

La préférence va au dimanche 28 et lundi 29 mai. La présidente tentera de faire coincider ces dates avec celles que choisiront l'Association canadienne des professeurs d'anglais (ACUTE) et l'Association canadienne de traductologie (ACT).

14. La présidente remercie, au nom de l'Association, l'Université d'Ottawa et le collège communautaire Red River pour l'aide que ces deux établissements ont apportée a la tenue du congrès (élaboration et impression du programme).

15. La séance est levée d $17 \mathrm{~h} 15$.

Jacqueline Bossé-Andrieu

Ron Blicq

Présidente de l'ACPRTS

Secrétaire de séance

suppléant 